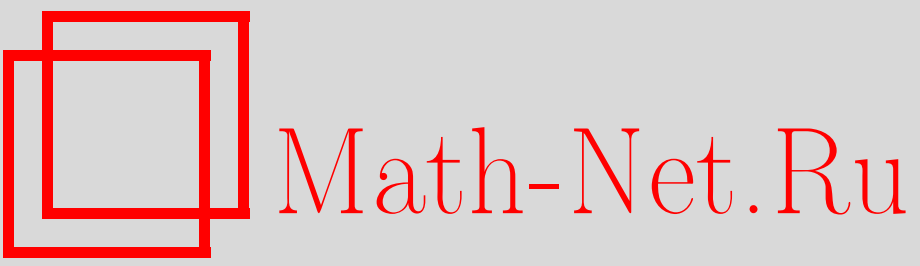

И. С. Ломов, Исследование сингулярно возмущенных и нерегулярно вырождающихся эллиптических задач, Итоги науки и техн. Сер. Соврем. мат. и ее прил. Темат. обз., 2021, том 191, 105-114

DOI: https://doi.org/10.36535/0233-6723-2021-191-105-114

Использование Общероссийского математического портала Math-Net.Ru подразумевает, что вы прочитали и согласны с пользовательским соглашением

http: //www.mathnet.ru/rus/agreement

Параметры загрузки:

IP : 54.205 .225 .156

26 апреля 2023 г., $13: 43: 43$ 


\title{
ИССЛЕДОВАНИЕ СИНГУЛЯРНО ВОЗМУЩЕННЫХ И НЕРЕГУЛЯРНО ВЫРОЖДАЮЩИХСЯ ЭЛЛИПТИЧЕСКИХ ЗАДАЧ
}

\author{
(c) 2021 г. И. С. ЛОМОВ
}

\begin{abstract}
АннотАция. На примере двух эллиптических задач показано, что метод регуляризации сингулярных возмущений, разработанный для построения регуляризованных асимптотических решений сингулярно возмущенных уравнений, может быть успешно применен к построению решений нерегулярно вырождающихся эллиптических задач.
\end{abstract}

Ключевые слова: сингулярное возмущение, спектр, вырождающееся эллиптическое уравнение, малый параметр, асимптотический ряд.

\section{INVESTIGATION OF SINGULARLY PERTURBED AND IRREGULARLY DEGENERATE ELLIPTIC PROBLEMS}

\author{
(c) 2021 I. S. LOMOV
}

\begin{abstract}
Using two elliptic problems as examples, we show that the method of regularization of singular perturbations developed for constructing regularized asymptotic solutions of singularly perturbed equations can be successfully applied to constructing solutions of irregularly degenerate elliptic problems.
\end{abstract}

Keywords and phrases: singular perturbation, spectrum, degenerate elliptic equation, small parameter, asymptotic series.

AMS Subject Classification: 35J75

Метод регуляризации сингулярных возмущений (см. $[2,3])$ позволяет получить регуляризованные асимптотические решения для широкого класса задач. При определенных условиях полученные асимптотические ряды по степеням малого параметра сходятся в обычном смысле. Мы покажем, что после небольшой модификации, этот метод также применим к решению граничных задач для нерегулярно вырождающихся эллиптических операторов. Мы назвали этот метод для эллиптических операторов методом спектрального описания особенностей (см. [3, гл. Х]). В обоих случаях спектр предельного оператора используется для описания особенностей решения.

1. Применение метода регуляризации к эллиптической задаче. Для эллиптических уравнений можно выделить круг сингулярно возмущенных задач в цилиндрических областях, при решении которых имеются две вязкие границы, не соприкасающиеся между собой. Применение метода регуляризации к таким задачам позволяет получить асимптотические ряды для решений. Рассмотрим простейшую задачу, с которой встречаются специалисты по электроразведке. Полагаем, что с глубиной $(z)$ проводимость среды не изменяется (это поможет нам определить в элементарных функциях собственные функции соответствующей задачи). Проводимость в перпендикулярном к глубине направлении $(y)$ изменяется медленно с изменением $y$. Задача двумерная. Для такой задачи удается написать аналитические приближенные решения. 
1.1. Постановка задачи. Для магнитной компоненты поля с помощью уравнений Максвелла ставится следующая задача:

$$
\begin{aligned}
& \frac{\partial^{2} u}{\partial y^{2}}+\frac{\partial^{2} u}{\partial z^{2}}-\kappa^{2}(\varepsilon y) u=0,\left.\quad u\right|_{z=0}=H,\left.\quad u\right|_{z=h}=0, \\
& \left.u\right|_{y=-M / \varepsilon}=H e^{-k_{1} z} \varphi_{1}(z),\left.\quad u\right|_{y=M / \varepsilon}=H e^{-k_{2} z} \varphi_{2}(z),
\end{aligned}
$$

где $H, M, k_{1}, k_{2}$ - постоянные, $\kappa^{2}(\varepsilon y)=-i \mu_{0} \omega \sigma(\varepsilon y)$, Re $\kappa>0, k_{1}^{2}=-i \mu_{0} \omega \sigma_{1}, k_{2}=-i \mu_{0} \omega \sigma_{2}$, $\operatorname{Re} k_{i}>0 ; \varepsilon$-малый положительный параметр, характеризующий медленное изменение проводимости $\sigma$ с изменением $y$. Если в указанной задаче сделать замену $\varepsilon y=x$, то новую задачу можно записать в виде

$$
\begin{gathered}
L_{\varepsilon} u \equiv \varepsilon^{2} \frac{\partial^{2} u}{\partial x^{2}}+\frac{\partial^{2} u}{\partial z^{2}}-\kappa^{2}(x) u=0,\left.\quad u\right|_{z=0}=H,\left.\quad u\right|_{z=h}=0, \\
\left.u\right|_{x=-M}=H e^{-k_{1} z} \varphi_{1}(z),\left.\quad u\right|_{x=M}=H e^{-k_{2} z} \varphi_{2}(z) .
\end{gathered}
$$

Нам удобнее иметь однородные условия по $z$. Поэтому введем замену искомой функции $v=$ $u-H(h-z) / h$. Тогда задача $(1),(2)$ примет свой окончательный вид

$$
\begin{gathered}
L_{\varepsilon} v \equiv \varepsilon^{2} \frac{\partial^{2} v}{\partial x^{2}}+\frac{\partial^{2} v}{\partial z^{2}}-\kappa^{2}(x) u=f(x, z),\left.\quad v\right|_{z=0}=\left.v\right|_{z=h}=0, \\
\left.v\right|_{x=-M}=q_{1}(z),\left.\quad v\right|_{x=M}=q_{2}(z),
\end{gathered}
$$

где $f(x, z)=H(h-z) / h, q_{1}(z)=H\left[e^{-k_{1} z} \varphi_{1}-(h-z) / h\right], q_{2}(z)=H\left[e^{-k_{2} z} \varphi_{2}-(h-z) / h\right]$. Решение задачи (3), (4) необходимо изучить при $\varepsilon \rightarrow 0$.

1.2. Регуляризачия и получение итерационных задач. Для решения задачи (3), (4) при $\varepsilon \rightarrow 0$ определяем спектр предельной однородной задачи:

$$
L_{0} \psi_{k} \equiv \frac{d^{2} \psi_{k}}{d z^{2}}-\kappa^{2}(x) \psi_{k}=\lambda_{k}(x) \psi_{k}(z, x),\left.\quad \psi_{k}\right|_{z=0}=\left.\psi_{k}\right|_{z=h}=0,
$$

т.е. находим $\lambda_{k}(x)=-\left(k^{2} \pi^{2}+h^{2} \kappa^{2}(x)\right) / h^{2}, \psi_{k}(z, x)=\sin (k \pi z / h), \operatorname{Re} \lambda_{k}=-k^{2} \pi^{2} / h^{2}<0$, $k=1,2, \ldots$ Будем предполагать выполненными следующие условия.

Условие А. Функции $\varphi_{1}(z), \varphi_{2}(z)$ таковы, что $q_{i}(z) \in C^{\infty}[0, h]$ и обеспечивают условия согласования в угловых точках прямоугольника $(-M \leqslant x \leqslant M) \times(0 \leqslant z \leqslant h)$ до любого порядка.

Отметим, что спектр оператора $L_{0}$ удовлетворяет следующим неравенствам $\ldots<\operatorname{Re} \lambda_{k}<\ldots<$ $\operatorname{Re} \lambda_{2}(x)<\operatorname{Re} \lambda_{1}(x)<0$.

Для регуляризации сингулярностей по $\varepsilon$ при $\varepsilon \rightarrow 0$ составим пучок операторов, отвечающий однородной части уравнения (3): $L(\lambda)=\varepsilon^{2} \lambda^{2} I+L_{0}$, где $I$-тождественный оператор. Чтобы определить спектр пучка, составим спектральное характеристическое уравнение: $\varepsilon^{2} \lambda^{2}+\lambda_{k}(x)=0$. Обозначим $\omega_{k}(x)=\sqrt{-\lambda_{k}(x)}, \operatorname{Re} \omega_{k}(x)>0$, и запишем спектр пучка в виде: $\lambda_{1 k}(x)=-\omega_{k}(x) / \varepsilon$, $\lambda_{2 k}(x)=\omega_{k}(x) / \varepsilon, k=1,2, \ldots$ По спектру пучка вводим счетное число дополнительных независимых переменных по формулам

$$
\tau_{k}=-\frac{1}{\varepsilon} \int_{-M}^{x} \omega_{k}(r) d r \equiv \varphi_{k}(x, \varepsilon), \quad t_{k}=\frac{1}{\varepsilon} \int_{M}^{x} \omega_{k}(r) d r \equiv g_{k}(x, \varepsilon), \quad k=1,2, \ldots
$$

Введем обозначения $\tau=\left(\tau_{1}, \tau_{2}, \ldots\right), t=\left(t_{1}, t_{2}, \ldots\right), \varphi(x, \varepsilon)=\left(\varphi_{1}, \varphi_{2}, \ldots\right), g(x, \varepsilon)=\left(g_{1}, g_{2}, \ldots\right)$.

Окончательная регуляризация задачи $(3),(4)$ производится следующим образом. Вместо искомого решения $v(x, z, \varepsilon)$ будем определять новую функцию $\tilde{v}(x, z, \tau, t, \varepsilon)$ такую, сужение которой совпадает с искомым решением, т.е.

$$
\left.\tilde{v}\right|_{\tau=\varphi, t=g} \equiv v(x, z, \varepsilon)
$$


Тогда из соотношения (6) с учетом выражений (5) получим

где

$$
\frac{\partial v}{\partial x}=\frac{\partial \tilde{v}}{\partial x}+\frac{1}{\varepsilon} D_{\omega} \tilde{v}
$$

$$
D_{\omega} \equiv \sum_{k=1}^{\infty} \omega_{k}(x)\left(\partial / \partial t_{k}-\partial / \partial \tau_{k}\right)
$$

$$
\frac{\partial^{2} v}{\partial x^{2}}=\frac{\partial^{2} \tilde{v}}{\partial x^{2}}+\frac{1}{\varepsilon}\left[2 D_{\omega}\left(\frac{\partial \tilde{v}}{\partial x}\right)+D_{\omega^{\prime}} \tilde{v}\right]+\frac{1}{\varepsilon^{2}} D_{\omega}^{2} \tilde{v}
$$

где

$$
D_{\omega}^{2} \equiv D_{\omega}\left(D_{\omega}\right), \quad D_{\omega^{\prime}} \equiv \sum_{k=1}^{\infty} \omega^{\prime}(x)\left(\partial / \partial t_{k}-\partial / \partial \tau_{k}\right)
$$

Поставим задачу для определения новой функции $\tilde{v}$, используя задачу (3), (4), соотношение (6) и выражение для второй производной. Получим

$$
\begin{gathered}
\tilde{L}_{\varepsilon} \tilde{v} \equiv \tilde{L}_{0} \tilde{v}+\varepsilon L_{1} \tilde{v}+\varepsilon^{2} L_{2} \tilde{v}=f(x, z),\left.\quad \tilde{v}\right|_{z=0}=\left.\tilde{v}\right|_{z=h}=0, \\
\tilde{v}(-M, z, 0, g(-M, \varepsilon), \varepsilon)=q_{1}(z), \quad \tilde{v}(M, z, \varphi(M, \varepsilon), 0, \varepsilon)=q_{2}(z),
\end{gathered}
$$

где $\tilde{L}_{0} \equiv D_{\omega}^{2}+L_{0}, L_{1} \equiv 2 D_{\omega} \partial / \partial x+D_{\omega^{\prime}}, L_{2} \equiv \partial^{2} / \partial x^{2}$. Для сокращения записей мы однородные краевые условия в (7) будем записывать как $\left.\tilde{v}\right|_{\Gamma_{1}}=0$, условие в точке $x=-M-$ как $\left.\tilde{v}\right|_{\Gamma_{2}}$, и в точке $x=M-$ соответственно $\left.\tilde{v}\right|_{\Gamma_{3}}$.

Задача (7), (8) уже регулярна по $\varepsilon$, поэтому ее решение будем определять в виде ряда по степеням $\varepsilon$ :

$$
\tilde{v}(x, z, \tau, t, \varepsilon)=\sum_{i=0}^{\infty} \varepsilon^{i} v_{i}(x, z, \tau, t) .
$$

Подставим этот ряд в задачу (7), (8) и приравняем коэффициенты при одинаковых степенях $\varepsilon$, обращаясь с функциями $g(-M, \varepsilon)$ и $\varphi(M, \varepsilon)$ как с функциями порядка $O(1)$ при $\varepsilon \rightarrow 0$. Мы получим следующие задачи для определения коэффициентов ряда (9):

$$
\begin{aligned}
& \tilde{L}_{0} v_{0}=f(x, z),\left.\quad v_{0}\right|_{\Gamma_{1}}=0,\left.\quad v_{0}\right|_{\Gamma_{2}}=q_{1},\left.\quad v_{0}\right|_{\Gamma_{3}}=q_{2}, \\
& \tilde{L}_{0} v_{1}=-L_{1} v_{0},\left.\quad v_{1}\right|_{\Gamma_{1}}=0,\left.\quad v_{1}\right|_{\Gamma_{2}}=0,\left.\quad v_{1}\right|_{\Gamma_{3}}=0 \\
& \tilde{L}_{0} v_{i}=-L_{1} v_{i-1}-L_{2} v_{i-2},\left.\quad v_{i}\right|_{\Gamma_{1}}=0,\left.\quad v_{i}\right|_{\Gamma_{2}}=0,\left.\quad v_{i}\right|_{\Gamma_{3}}=0, \quad i=2,3, \ldots
\end{aligned}
$$

1.3. Однозначная разрешимость итерачионных задач. Для обоснования разрешимости итерационных задач (10)-(12) рассмотрим пространство $U$ функций, представимых равномерно сходящимися по $x$ и $z$ рядами по собственным функциям оператора $\tilde{L}_{0}$ (при каждом фиксированном $x$ ):

$$
\begin{aligned}
& U=\{u(x, z, \tau, t): u= \sum_{k=1}^{\infty}\left[u_{k}(x) e^{\tau_{k}}+u_{k k}(x) e^{t_{k}}+\widehat{u}_{k}(x)\right] \sin \frac{k \pi z}{h}, \\
&\|u\|^{2}=\sum_{k=1}^{\infty}\left(\left|u_{k}(x)\right|^{2}+\left|u_{k k}(x)\right|^{2}+\left|\lambda_{k}\right|^{2}\left|\widehat{u}_{k}(x)\right|^{2}\right)<+\infty \\
&\left.-\infty \leqslant \operatorname{Re} \tau_{k} \leqslant 0,-\infty \leqslant \operatorname{Re} t_{k} \leqslant 0 ; u_{k}(x), u_{k k}(x), \widehat{u}_{k}(x) \in C^{\infty}[-M, M]\right\} .
\end{aligned}
$$

Определение 1. Назовем пространство $V=\left\{v(x, z, \tau, t): \partial^{m} v / \partial x^{m} \in U, m=0,1,2, \ldots\right\}$ nространством безрезонансных решений.

Скалярное произведение определим следующим образом:

$$
\forall v, r \in V: \quad(v, r)=\sum_{k=1}^{\infty}\left(v_{k}(x) \bar{r}_{k}(x)+v_{k k}(x) \bar{r}_{k k}(x)+\widehat{v}_{k}(x) \overline{\hat{r}}_{k}(x)\right) \quad \forall x \in[-M, M] .
$$


Нетрудно показать, что $\tilde{L}_{0}^{*}=L_{0}^{*}+D_{\bar{\omega}}$ и что базисными элементами ядра оператора $L_{0}^{*}$ в $V$ являются функции

$$
\left\{u_{k}=e^{\tau_{k}} \sin \frac{k \pi z}{h}\right\}_{1}^{\infty}, \quad\left\{v_{k}=e^{t_{k}} \sin \frac{k \pi z}{h}\right\}_{1}^{\infty} .
$$

Обозначим объединение этих последовательностей через $\left\{w_{j}\right\}=\left\{u_{i}\right\} \cup\left\{v_{k}\right\}$. Имеет место следующая теорема о нормальной разрешимости уравнения

$$
\tilde{L}_{0} v=\theta(x, z, \tau, t)
$$

в пространстве безрезонансных решений $V$.

Теорема 1. Если в уравнении (13) правая часть $\theta \in V$ и спектр оператора $L_{0}$ при каждом $x$ удовлетворяет неравенству $\operatorname{Re} \omega_{k}(z)>0, k=1,2, \ldots$, то для разрешимости уравнения (13) в пространстве $V$ необходимо и достаточно, чтобы правая часть $\theta$ была ортогональна ядру оператора $\tilde{L}_{0}^{*}($ тождественно по $x \in[-M, M])$.

Имеет место теорема о единственности решения в пространстве $V$ каждой из задач $(10),(11)$.

Теорема 2. Пусть выполнены условия теоремы 1 и правая часть $\theta \perp \operatorname{Ker} \tilde{L}_{0}^{*}$ (равномерно по $x)$. Тогда решение уравнения (13) при условиях

$$
\left.v\right|_{\Gamma_{i}}=q_{i}(z), \quad i=1,2,3, \quad\left(q_{1}(z) \equiv 0\right), \quad\left(L_{1} v, w_{j}\right) \equiv 0, \quad j=1,2, \ldots,
$$

существует для достаточно малых $\varepsilon>0$ и единственно в пространстве $V$.

Теоремы 1 и 2 доказываются так же, как и аналогичные теоремы в [2]. С помощью этих теорем устанавливается однозначная разрешимость задач (10)-(12).

1.4. Теорема об асимптотической сходимости ряда. Ряд (9) получен как формальное решение задачи (7), (8). Имеет место следующая лемма о соотношении ряда (9) с решением исходной задачи (3), (4).

Лемма 1. Сужение $\left.\tilde{v}\right|_{\tau=\varphi, t=g}$ ряда (9) является формальным асимптотическим рядом для решения задачи (3), (4).

Введем обозначения

$$
v_{\varepsilon N}(x, z, \tau, t)=\sum_{i=0}^{N} \varepsilon^{i} v_{i}(x, z, \tau, t), \quad u_{\varepsilon N}(x, z, \tau, t)=v_{\varepsilon N}(x, z, \tau, t)+H(h-z) / h .
$$

Имеет место следующая теорема об оценке остаточного члена.

Теорема 3. Пусть выполняются условия теоремы $1, \kappa(x) \in C^{\infty}[-M, M]$, и вышеописанным методом получен ряд (9). Тогда сужение ряда (9) при $\tau=\varphi(x, \varepsilon), t=g(x, \varepsilon)$ является асимптотическим рядом для решения задачи (1), (2), т.е. существует такое $\varepsilon_{0}>0$, что при $0<\varepsilon<\varepsilon_{0}$ имеет место неравенство

$$
\left\|u(x, z, \varepsilon)-u_{\varepsilon N}(x, z, \varphi(x, \varepsilon), g(x, \varepsilon))\right\|_{L_{2}(D)} \leqslant K \varepsilon^{N+1}, \quad N=0,1,2, \ldots,
$$

где и( $x, z, \varepsilon)$ - решение задачи (1), (2), постоянная $K$ не зависит от $\varepsilon ; D$ - прямоугольник, в котором решается задача (1), (2). Сужение ряда (9), т.е. ряд

$$
\sum_{i=0}^{\infty} \varepsilon^{i} v_{i}(x, z, \varphi(x, \varepsilon), g(x, \varepsilon))
$$

с $v_{i} \in V$, единственно. 
1.5. Главный член асимптотики. В заключение остановимся на особенностях главного члена асимптотики решения изучаемой задачи (1), (2). Он имеет вид

$$
\begin{aligned}
& u_{\varepsilon 0}=\frac{H\left(e^{2 \kappa h}-e^{2 \kappa z}\right) e^{-\kappa z}}{e^{2 \kappa h}-1}+ \\
& +\sqrt{h} \sum_{k=1}^{\infty} \frac{1}{\sqrt[4]{\kappa^{2} \pi^{2}+h^{2} \kappa^{2}(x)}}\left(c_{k} \exp \left(-\frac{1}{\varepsilon} \int_{-M}^{x} \omega_{k}(r) d r\right)+r_{k} \exp \left(\frac{1}{\varepsilon} \int_{M}^{x} \omega_{k}(r) d r\right)\right) \sin \frac{k \pi z}{h}
\end{aligned}
$$

и обладает следующими свойствами:

(1) удовлетворяет краевым условиям по z точно и с точностью до экспоненциально малых слагаемых краевым условиям (2);

(2) уравнению(1) удовлетворяет с точностью до членов, содержащих $\varepsilon^{2}$, т.е. $L_{\varepsilon} u_{\varepsilon 0} \equiv \varepsilon^{2} F(x, z, \varepsilon)$, где $\|F(x, z, \varepsilon)\|$ равномерно ограничена по $\varepsilon$. Функцию $F$ можно выписать:

$$
\begin{aligned}
F=-H \frac{\partial^{2}}{\partial x^{2}}\left[\frac{\left(e^{2 \kappa(x) h}-e^{2 \kappa(x) z}\right) e^{-\kappa(x) z}}{e^{2 \kappa(x) h}-1}\right]+ \\
+\frac{h}{2} \sum_{k=1}^{\infty} \frac{d_{k}}{\left(\kappa^{2} \pi^{2}+h^{2} \kappa^{2}(x)\right)^{9 / 4}}\left(c_{k} \exp \left(-\frac{1}{\varepsilon} \int_{-M}^{x} \omega_{k}(r) d r\right)+r_{k} \exp \left(\frac{1}{\varepsilon} \int_{M}^{x} \omega_{k}(r) d r\right)\right)
\end{aligned}
$$

где $c_{k}=\alpha_{k} \sqrt{\omega_{k}(-M)}, r_{k}=\beta_{k} \sqrt{\omega_{k}(M)}, \alpha_{k}=\kappa h^{2}\left(\frac{3}{2} \kappa^{2}+\kappa^{2} \kappa^{\prime \prime}\right)-\kappa^{2} \pi^{2}\left(\kappa^{\prime 2}+\kappa \kappa^{\prime \prime}\right)$. Следовательно, мы получили очень хорошее приближение для практических расчетов и для анализа решения задачи (1), (2).

Можно отметить, что ряд (15) сходится вместе с производными. Поэтому при анализе решения вместо ряда можно брать конечное число слагаемых. Внутри области решение задачи (1) описывается в основном первым слагаемым в силу того, что обе экспоненты ряда малы по модулю. Однако экспоненты на границах (одна - на одной, другая - на другой) и вблизи границы $(x= \pm M)$ не малы; и именно за счет наличия этого ряда в решении выполнены краевые условия при $x= \pm M$. Таким образом, пограничный слой в рассмотренной задаче описывается двумя счетными наборами экспонент, каждый из которых существенно отличен от нуля только на соответствующей границе.

Метод разработан для широкого класса краевых и смешанных задач, как линейных, так и нелинейных.

2. Вырождающиеся эллиптические дифференциальные операторы. Классическая теорема Коши-Ковалевской гарантирует локальную аналитичность решения линейного дифференциального уравнения, представленного в нормальной форме, если его коэффициенты, правая часть и начальные данные аналитичны (в случае вещественных переменных - разлагаются в сходящиеся степенные ряды). Исследования вырождающихся эллиптических уравнений показали, что и в этом случае аналитичность коэффициентов уравнения наследуется его решением. Фундаментальная система состоит из функций, представляющих собой произведение голоморфной в окрестности точки (плоскости) вырождения функции на функцию, которая может иметь особенность на указанном множестве. Эта особенность либо степенная, при этом показатель находится по коэффициентам уравнения, либо степенная и логарифмическая. Для обоснования существования решения краевой задачи для вырождающихся уравнений, как правило, используют неявные методы построения решения (например, метод барьеров). При этом затруднительно выяснить структуру решения и проследить, как аналитичность коэффициентов и правой части уравнения отражается на решении задачи.

Здесь мы на примере модельной задачи с нерегулярно вырождающимся эллиптическим оператором, заданным в прямоугольнике, с аналитическими данными по переменной вырождения, предложим метод решения краевой задачи для неоднородного уравнения. Покажем, как результат теоремы Коши-Ковалевской переносится на этот класс задач. 
Мы также покажем, что особенности решения можно описать в соответствии с методом регуляризации сингулярных возмущений (подобно случаю, когда коэффициент при старшей производной $y^{2}$ заменен на $\varepsilon$ - малый параметр). Как и в методе регуляризации для описания особенностей решения привлекается спектр предельного (особого) оператора - оператора на линии вырождения, при этом каждое собственное значение участвует в описании особенности. Наглядно просматривается структура решения и, в частности, его зависимость от переменной вырождения. Приведем пример задачи, для которой выполнены все условия сформулированных утверждений.

В прямоугольнике $D=(0<x<1) \times(0<y<b)$ требуется найти ограниченное в окрестности линии $y=0$ решение задачи

$$
\begin{gathered}
y^{2} u_{y y}+u_{x x}-a^{2}(y) u=f(x, y), \quad(x, y) \in D, \\
\left.u\right|_{x=0}=\left.u\right|_{x=1}=\left.u\right|_{y=b}=0, \quad|u(x, 0)|<\infty,
\end{gathered}
$$

где $u(x, y) \in C(\bar{D}) \cap C^{2}(D), a^{2}(y) \geqslant 0$, функции $a^{2}(y)$ и $f(x, y)$ аналитические в точке $y=0$ (при каждом $x \in G=(0,1))$ и разлагаются в ряды по степеням $y ; f \in C(D)$. Решение удовлетворяет всем условиям задачи в классическом смысле (частный случай задачи Е по терминологии М. В. Келдыша).

2.1. Построение формального решения. Приведем схему построения формального решения задачи (16). Затем приведем условия, при которых оно является классическим решением.

Обозначим $a^{2}(0)=a_{0}$ и $a^{2}(y)-a_{0}=a_{1}(y)$ и разделим переменные в задаче. Положим $u(x, y)=X(x) Y(y)$. Подставим это выражение в однородное уравнение (16) и разделим его на $X Y$ :

$$
y^{2} \frac{Y^{\prime \prime}}{Y}-a_{1}(y)=a_{0}-\frac{X^{\prime \prime}}{X}=\lambda .
$$

Для $X$ имеем задачу на собственные функции для предельного (особого) оператора $L$, который получается из левой части (16) при $y=0$ :

$$
X^{\prime \prime}+\left(\lambda-a_{0}\right) X=0, \quad X(0)=X(1)=0 .
$$

Обозначим собственные значения $\mu_{k}=-\lambda_{k}^{2}$ и собственные функции $\psi_{k}(x)$ и положим

$$
\lambda_{k}^{2}=a_{0}+k^{2} \pi^{2}, \quad \psi_{k}(x)=\sqrt{2} \sin \pi k x, \quad k \in \mathbb{N} .
$$

Для выделения особенности по $y$ отбросим подчиненный член $a_{1} Y$ и рассмотрим уравнение Эйлера $y^{2} Y^{\prime \prime}-\lambda_{k}^{2} Y=0$. Положив $Y=y^{\alpha}$, получим определяющее уравнение

$$
\alpha(\alpha-1)-\lambda_{k}^{2}=0
$$

его корни имеют вид

$$
\alpha_{1,2}=\frac{1 \pm \sqrt{1+4 k^{2}}}{2} .
$$

Чтобы в решении не появилось логарифмических особенностей, потребуем, чтобы $\alpha_{1}-\alpha_{2} \notin \mathbb{Z}$, т.е.

$$
\lambda_{k}^{2} \neq \frac{n^{2}-2}{4} \quad \forall k, n \in \mathbb{N}
$$

(тогда $\lambda_{k}^{2} \neq l(l-1)$ для всех $\left.l, k \in \mathbb{N}\right)$. Условие (17) можно переписать в терминах коэффициента $a^{2}$ как $a^{2}(0) \neq\left(n^{2}-1\right) / 4-k^{2} \pi^{2}$ при всех $k, n \in \mathbb{N}$. Учитывая условие $|u(x, 0)|<\infty$, оставляем только положительный корень, который обозначим $r_{k}=\left(1+\sqrt{1+4 \lambda_{k}^{2}}\right) / 2$.

По аналогии с методом регуляризации сингулярных возмущений введем счетное число новых переменных $\tau_{k} \in[0,1], k \in \mathbb{N}$, и положим

$$
\tau=\left(\tau_{1}, \tau_{2}, \ldots\right), \quad g_{k}\left(y^{r_{k}}\right)=(y / b)^{r_{k}}, \quad g\left(y^{r_{k}}\right)=\left(g_{1}, g_{2}, \ldots\right), \quad \sum_{k}=\sum_{k=1}^{\infty} .
$$

Ищем решение задачи (16) в виде ряда

$$
v(x, y, \tau)=\sum_{k}\left[\tau_{k} \varphi_{k}(y)+\eta_{k}(y)\right] \psi_{k}(x),
$$


где $\varphi_{k}$ и $\eta_{k}$ - аналитические функции, представимые рядами по степеням $y$ на $[0, b]$ и $\left.v(x, y, \tau)\right|_{\tau=g\left(y^{r} k\right)} \equiv u(x, y)$, т.е. указанное сужение функции $v$ является решением задачи (16).

Поставим задачу для функции $v$. Поскольку

$$
u_{y}=\left.\left(v_{y}+v_{\tau} \tau_{y}\right)\right|_{\tau=g\left(y^{r} k\right)}=v_{y}+\frac{1}{y} \sum_{k} r_{k} \tau_{k} v_{\tau_{k}},
$$

получаем из (16), что

$$
\begin{gathered}
y^{2} v_{y y}+2 y \sum_{k} r_{k} \tau_{k} v_{y \tau_{k}}+\sum_{k} r_{k}\left(r_{k}-1\right) \tau_{k} v_{\tau_{k}}+v_{x x}-a^{2}(y) v=f(x, y), \\
(x, y) \in G, \quad \tau_{k} \in G, \quad k \geqslant 1, \\
\left|v\left(x, 0+, \tau_{k}\right)\right|<\infty,\left.\quad v\right|_{x=0}=\left.v\right|_{x=1}=\left.v\right|_{y=b, \quad \tau_{k}=1}=0 \\
\left.v(x, y, \tau)\right|_{\tau=g\left(y^{r}\right)}=u(x, y) .
\end{gathered}
$$

Задача (19) более широкая, чем (16), но можно построить ее решение (18) без особенности по $y$; положив $\tau=g\left(y^{r_{k}}\right)$, мы получим решение задачи (16). По аналогии с теорией малого параметра можно назвать задачу (16) сингулярно возмущенной, а (19) - регулярно возмущенной, так как предельный оператор в (19) (при $y=0$ ) допускает удовлетворение всем краевым условиям, в том числе и при $y=b, \tau_{k}=1$, т.е. любое решение предельной задачи в (19) принадлежит области определения рассматриваемого оператора в (19).

Система $\left\{\psi_{k}\right\}$ образует ортонормированный базис в $\mathcal{L}^{2}(G)$, поэтому

$$
f(x, y)=\sum_{k} f_{k}(y) \psi_{k}(x) .
$$

Подставив (18) в (19), получим задачи для $\varphi_{k}(y)$ и $\eta_{k}(y)$ :

$$
\begin{gathered}
y^{2} \eta_{k}^{\prime \prime}-\left[\lambda_{k}^{2}+a_{1}(y)\right] \eta_{k}=f_{k}(y), \quad y \in(0, b), \quad\left|\eta_{k}(0)\right|<\infty, \quad k \in \mathbb{N}, \\
\eta_{k}=\sum_{l=0}^{\infty} \eta_{k}^{l} y^{l}, \quad a^{2}(y)=\sum_{l=0}^{\infty} a^{l} y^{l}, \quad f_{k}(y)=\sum_{l=0}^{\infty} f_{k}^{l} y^{l}, \quad y \in(-R, R), \quad R \geqslant b ; \\
y^{2} \varphi_{k}^{\prime \prime}+2 y r_{k} \varphi_{k}^{\prime}-a_{1}(y) \varphi_{k}=0, \quad y \in(0, b), \quad\left|\varphi_{k}(0)\right|<\infty, \\
\varphi_{k}(b)=-\eta_{k}(b), \quad \varphi_{k}(y)=\sum_{l=0}^{\infty} \varphi_{k}^{l} y^{l}, \quad y \in(-R, R), \quad k \in \mathbb{N},
\end{gathered}
$$

Решая последовательно задачи (20)-(22), получаем формальное решение задачи (19). Приведем условия, при которых задачи (20) и (22) разрешимы.

\section{2. Формулировка результатов.}

Теорема 4. Пусть функиия $a^{2}(y)$ принадлежит $\mathcal{L}(0, b)$ и разлагается в ряд (21) при $R=b$; пусть справедливо условие $(17), f(x, y) \in \mathcal{L}(D)$ и $f(x, y) \in \mathcal{L}_{x}^{2}(G)$ для калсдого у $\in[0, b)$. Тогда для того чтобы задача (19) имела формальное и притом единственное решение вида (18), необходимо и достаточно, чтобы функиии $f_{k}(y)$ разлагались в рлды (21) при $R=b$.

Необходимость условия теоремы доказывается подстановкой (18) в (19) и приравниванием коэффициентов при $\psi_{k}$. Достаточность вытекает из следующих утверждений.

Лемма 2. Пусть коэфбициент $a^{2}(y)$ представим в виде сходящегося ряда $(21), R=b$. Уравнение (20) имеет единственное аналитическое решение на $(-b, b)$ при каждом $k \in \mathbb{N}$ тогда и только тогда, когда его правая часть $f_{k}(y)$ является аналитической функцией на $(-b, b)$, т.е. может быть представлена рядом (21), а также выполняется условие $\lambda_{k}^{2} \neq l(l-1)$ для всех $l, k \in \mathbb{N}$. Если при этом $f_{k}(y), a^{2}(y) \in \mathcal{L}(0, b)$, то существуют конечные предельные значения $\eta_{k}^{(\alpha)}(b-0), \alpha=0,1$. 
Лемма 3. Пусть выполняется условие теоремы 4 относительно $a^{2}$, справедливо $f_{k}(y) \in$ $\mathcal{L}(0, b)$ и пусть $\lambda_{k}^{2} \neq l(l-1)$ для всех $k, l \in \mathbb{N}$. Тогда задача $(22)$ имеет решение, и притом единственное. Это решение имеет конечные предельные значения $\varphi_{k}^{(\alpha)}(b-0), \alpha=0,1$.

Отметим, что из условия $f \in \mathcal{L}(D)$ следует выполнение условия $f_{k} \in \mathcal{L}(0, b)$.

Для того чтобы доказать, что формальное решение задачи (19) является его классическим (регулярным) решением, нужно получить оценки решений задач $(20)$ и $(22)$ по $k$. Сформулируем соответствующие утверждения в виде двух лемм.

Лемма 4. Пусть следующие условия (условия A1) выполняются при некотором фиксированном числе $R>b$ :

(1) степенные ряды (21) для $a^{2}(y)$ и $f_{k}(y)$ сходятся на $(-R, R)$;

(2) существуют такие $M=$ const $>0 u R_{1} \in(b, R)$, что $\left|f_{k}^{l}\right| \leqslant M / R_{1}^{l}$ для всех $l \geqslant 0, k \geqslant 1$;

(3) оценка малых знаменателей: пусть

$$
\beta_{k}^{l} \equiv l(l-1)-\lambda_{k}^{2}, \quad l \geqslant 0, \quad k \geqslant 1, \quad \delta_{k} \equiv \min _{l}\left|\beta_{k}^{l}\right|=\left|\beta_{k}^{J}\right|>0 ;
$$

найдутся такие постоянные $k_{1} \in \mathbb{N}, c_{\delta} \in \mathbb{R}_{+} u q_{0} \in\left(b / R_{1}, R / b\right)$ что $\delta_{k} \geqslant c_{\delta} J^{2} q_{0}^{J}$ для всех $k \geqslant k_{1}$.

Тогда для решения задачи (20), (21) справедливы оченки

$$
\eta_{k}^{(\alpha)}(y)=O\left(1 / k^{2}\right), \quad \eta_{k}^{(\alpha)}(y)=-(\pi k)^{-2}+O\left(1 / k^{4}\right), \quad \alpha=0,1,2,
$$

равномерно по $y \in[-b, b]$, начиная с некоторого номера $k=k_{0}$.

Отсюда вытекает следующее утверждение.

Следствие 1. Пусть выполняются условия $\mathbf{A 1}$ и ряд Фуръе функиии $f(x, y)$ по системе $\psi_{k}(x)$ сходится равномерно по $x \in[c, d]$ для всех $[c, d] \subset G$ при каждом $y \in[0, b)$. Тогда ряд

$$
\sum_{k} \eta_{k}(y) \psi_{k}(x)
$$

допускает двукратное почленное дифференцирование по х и у в D.

Лемма 5. Пусть выполняются условия А1. Тогда для решения задачи (22) имеют место оценки

$$
\varphi_{k}(y)=(\pi k)^{-2} f_{k}(b)\left(1+O\left(k^{-1}\right)\right), \quad \varphi_{k}^{(\alpha)}=k^{-2} f_{k}(b) O\left(k^{-1}\right), \quad \alpha=1,2,
$$

равномерно по $y \in[0, b)$, начиная с некоторого номера $k=k_{0}$.

Следствие 2. Пусть выполняются условия $\mathbf{A 1}, f \in \mathcal{L}_{x}^{2}(G)$ при всех $y \in[0, b)$, ряд Фуръе функиии $f(x, y)$ по системе $\psi_{k}(x)$ сходится равномерно по $x \in[c, d]$ для любого $[c, d] \subset G$ при каждом $y \in[0, b)$ и пусть $\left\{\tau_{k}\right\}$ - монотонная последовательность. Тогда ряд

$$
\sum_{k} \tau_{k} \varphi_{k}(y) \psi_{k}(x)
$$

допускает двукратное почленное дифберениирование по $x$ и у в $D$; при этом ряд, содержащий $\psi_{k}^{\prime \prime}$ сходится в D, а все остальные ряды сходятся абсолютно и равномерно в $\bar{D}$.

Оператор в (19) содержит два ряда: с функциями $v_{y \tau_{k}}$ и $v_{\tau_{k}}$. Исследуем их на сходимость.

Следствие 3. Пусть выполняются условия следствия 2. Тогда ряд

$$
\sum_{k} r_{k} \tau_{k} v_{y \tau_{k}}
$$

сходится абсолютно и равномерно в $\bar{D}$, а ряд

$$
\sum_{k} r_{k}\left(r_{k}-1\right) \tau_{k} v_{\tau_{k}}
$$

сходится в D. 
Теорема 5. Пусть выполняются условия A1, условие (17), пусть $f \in \mathcal{L}_{x}^{2}(G)$ при всех у $\in$ $[0, b)$, пусть ряд Фурве функиии $f(x, y)$ по системе $\psi_{k}(x)$ сходится равномерно по $x \in[c, d]$ при всех $[c, d] \subset G$ при каждом $y \in[0, b)$, пусть $f(x, y) \in \mathcal{L}_{y}(0, b)$ при всех $x \in G$, и пусть $\left\{\tau_{k}\right\}$ - монотонная последовательность. Тогда формальное решение (18) задачи (19) будет ее классическим решением.

Теорема 6. Пусть выполняются условия (1) и (3) из условий A1, пусть выполняется условие (17), $\left\{\tau_{k}\right\}$ - монотонная последовательность, пусть ряд

$$
f(x, y)=\sum_{l=0}^{\infty} f^{l}(x) y^{l}
$$

сходится по у на $(-R, R)$ при всех $x \in G$ и сходится в среднем по $x \in G$ при всех $y \in(-R, R)$, пусть ряд (21) для $f_{k}(y)$ сходится равномерно по $k \in \mathbb{N}, f(x, y) \in \mathcal{L}_{x}^{2}(G)$, пусть ряд

$$
f(x, y)=\sum_{k} f_{k}(y) \psi_{k}(x)
$$

сходится равномерно по $x$ на любом отрезке $[c, d] \subset G$ при каждом $y \in[0, b)$, и пусть

$$
\forall L \in \mathbb{N} \exists c=\text { const }>0:\left|f_{k}^{l}\right| \leqslant c \partial \Omega, 0 \leqslant l \leqslant L u k \geqslant 1 .
$$

Тогда задача (19) имеет и притом единственное классическое решение, представимое в виде ряда (18), сужение которого при $\tau=g\left(y^{r_{k}}\right)$ является решением задачи (16).

Замечание 1. Условие (23) и требование равномерной сходимости ряда $f_{k}(y)$ по $k$ обеспечивают выполнение условия 2 из условий $\mathbf{A 1}$. Для выполнения условия (23) достаточно, чтобы удовлетворялось условие $\partial^{l} f(x, 0) / \partial y^{l} \in \mathcal{L}(G), l \geqslant 1$; при этом коэффициенты Фурье

$$
f_{k}^{l}=\int_{\bar{G}} f^{l}(x) \psi_{k}(x) d x \rightarrow 0, \quad k \rightarrow \infty,
$$

и условие (23) выполняется для каждого конечного числа $L$ (здесь $f^{l}(x)$ - коэффициенты разложения функции $f$ в ряд Тейлора).

Замечание 2. Сходимость в среднем степенного ряда для $f(x, y)$ требуется для того, чтобы функция $f_{k}(y)$ была представима степенным рядом.

Замечание 3. Если $f(x, y) \in W_{2}^{1}(G)$ по $x$ при всех $y \in(0, b)$, то $\eta_{k}(y)=O\left(k^{-3}\right)$, как и в рассмотренном ниже примере. При этом ряд Фурье функции $f$ по системе $\left\{\psi_{k}\right\}$ сходится равномерно по $x$ при всех $[c, d] \subset(0,1)$ при каждом $y$.

Замечание 4. Коэффициент $a^{2}$ в уравнении (16) может зависеть от $x$. В частности, если

$$
a^{2}(x, y)=a^{2}(x, 0)+\sum_{l=1}^{\infty} a_{l} y^{l}, \quad a_{l}=\text { const },
$$

то все результаты, приведенные выше, остаются в силе. Эти результаты переносятся и на случай, когда предельный оператор $L$ задачи $(16)$ - это общий линейный нагруженный дифференциальный оператор с переменными коэффициентами и общими краевыми условиями, задаваемыми интегралами Стилтьеса. Предполагается, что $L$ удовлетворяет условиям безусловной базисности в $\mathcal{L}^{2}(G)$. Функции $\psi_{k}(x)$ вместе со своими производными могут иметь разрывы первого рода в счетном числе точек отрезка $[0,1]$. В (16) производную $u_{x x}$ можно заменить на $\Delta u$-оператор Лапласа по переменным $x, z, \ldots$.

Замечание 5. Возвращаясь к теореме Коши-Ковалевской об аналитичности решения линейного дифференциального уравнения, представленного в нормальной форме, заметим, что и в рассмотренной задаче (16) решение наследует свойство аналитичности коэффициентов и правой части уравнения. Решение (18) - это сумма слагаемых, каждое из которых состоит из двух частей: аналитической функции $\eta_{k}(y) \psi_{k}(x)$ по $y$ и произведения аналитической функции $\varphi_{k}(y) \psi_{k}(x)$ по $y$ на функцию $y^{r_{k}}$, описывающую особенность уравнения. 
Пример. Пусть $a^{2}(y)=y^{2}$ и $f(x, y)=x y$ в задаче (16). Тогда

$$
\lambda_{k}^{2}=\pi^{2} k^{2}, \quad f_{k}(y)=(-1)^{k+1} \sqrt{2}(\pi k)^{-1} y,
$$

и ряд Фурье

$$
f(x, y)=y \frac{2}{\pi} \sum_{k} \frac{(-1)^{k+1}}{k} \sin \pi k x
$$

сходится равномерно на каждом отрезке $[c, d] \subset(0,1)$ при всех $y \in[0, b)$. Для решения $\eta_{k}(y)$ задачи (20) получаем ряд

$$
\eta_{k}(y)=\frac{(-1)^{k} \sqrt{2}}{(\pi k)^{3}} y\left\{1+\sum_{l=1}^{\infty} y^{2 l} \prod_{j=1}^{l}\left[2 j(2 j+1)-\pi^{2} k^{2}\right]^{-1}\right\},
$$

имеет место оценка $\eta_{k}(y)=O\left(1 / k^{3}\right)$, и малые знаменатели отделены от нуля величиной

$$
\min _{j}\left|2 j(2 j+1)-\pi^{2} k^{2}\right|>\frac{c_{0}}{k^{\Lambda}}>c_{0} J^{2} q_{0}^{J}
$$

для всех $k>k_{1}>1$, где $0<\Lambda \leqslant 16, q_{0}<1$ - некоторая постоянная, и $J \sim \pi k$.

Для решения $\varphi_{k}(y)$ задачи (22) получим ряд

$$
\begin{gathered}
\varphi_{k}(y)=\varphi_{k}^{\circ}\left\{1+\sum_{l=1}^{\infty} y^{2 l}\left[(2 l) ! ! \prod_{j=1}^{l}\left(2 r_{k}+2 j-1\right)\right]^{-1}\right\}, \\
\varphi_{k}^{\circ}=-\eta_{k}(b)\left\{1+\sum_{l=1}^{\infty} b^{2 l}\left[(2 l) ! ! \prod_{j=1}^{l}\left(2 r_{k}+2 j-1\right)\right]^{-1}\right\}, \\
\varphi_{k}(y)=(-1)^{k} b \sqrt{2} \frac{1}{(\pi k)^{3}}\left[1+O\left(\frac{1}{k}\right)\right] .
\end{gathered}
$$

Все условия теорем 5 и 6 выполнены, решение задачи (16) имеет вид (18) с коэффициентами $\varphi_{k}$ и $\eta_{k}$, выписанными выше, $\tau_{k}=(y / b)^{r_{k}}$.

\section{СПИСОК ЛИТЕРАТУРЫ}

1. Емелъянов Д. П., Ломов И. С. Построение точных решений нерегулярно вырождающихся эллиптических уравнений с аналитическими коэффициентами// Диффер. уравн. - 2019. - 55, № 1. - С. 45-58.

2. Ломов $C$. A. Введение в общую теорию сингулярных возмущений. - М.: Наука, 1981.

3. Ломов С. А., Ломов И. С. Основы математической теории пограничного слоя. - М.: Изд-во МГУ, 2011.

Ломов Игорь Сергеевич

Московский государственный университет им. М. В. Ломоносова

E-mail: lomov@cs.msu.ru 